\title{
A Novel, Photosynthesis-Associated Thioredoxin-like Gene Final Technical Report
}

\section{Background}

In 1995, Collier and Grossman reported the discovery of a novel gene, designated $t x l A$, in the obligately photoautotrophic cyanobacterium Synechococcus sp. strain PCC 7942. The protein predicted to be encoded by this gene consists of an $\mathrm{N}$-terminal signal-sequence-like region, a central domain with similarity to thioredoxins, and a unique hydrophilic $\mathrm{C}$-terminal domain. Although it was not possible to inactivate $t x l A$ in the thioredoxin-like domain (that is, it is essential for growth), it was possible to generate mutants of PCC 7942 that lacked only the Cterminal domain of TxlA, retaining the $\mathrm{N}$-terminal and thioredoxin-like domains intact. These mutants exhibited phenotypes suggesting that the relative abundance and functional relationships of their photosynthetic complexes were altered. The precise defect in the mutant was not identified, so it is not known whether the observed phenotypes represent the primary effects of truncation of TxlA or secondary effects. In the time since this proposal was submitted, the homologue of txlA in Arabidopsis thaliana, hcf164, has been investigated (Lennartz et al. 2001). HCF164 had been identified in the original proposal as a homologue of TxlA, 'PDO At'. HCF164' mutant plants could be grown heterotrophically, but appeared to completely lack cytochrome $b_{6} f$ complex. The authors suggested that HCF164 was required for the biogenesis of cytochrome $f$, a $c$-type cytochrome (with a covalently attached heme). Other thioredoxin-like proteins are known to be involved in the attachment of heme to $c$-type cytochromes.

Homologues of TxlA and HCF164 can be found only in oxygenic phototrophs (there are now over 40, mainly from cyanobacterial genome sequences and plant EST databases), and not in any other organisms with complete genome sequences. Combined with the phenotypic data on TxlA and HCF164 mutants, this observation suggests to us that TxlA and its homologues affect some aspect of the structure and/or function of the photosynthetic apparatus; the proposed role of HCF164 in cytochrome $f$ synthesis would fit this expectation. A thioredoxinlike protein such as TxlA might also be involved in the maturation of one or more proteins containing disulfide bonds, or function as a redox status sensor, signal transducer, or regulator involved in altering the structure and/or function of the photosynthetic apparatus to match environmental conditions.

Specific hypotheses to be tested during this project were:

1. TxlA is redox active, and the two putative thioredoxin active-site cysteine residues are required for its redox activity.

The first part of this hypothesis was tested by producing and assaying recombinant TxlA and Sll1980 in vitro. Not surprisingly, with the N-terminal hydrophobic domain, both were insoluble. However, with the N-terminal extension removed, both were soluble and demonstrated thioredoxin-like redox activity in two standard assays: insulin reduction and DTNB reduction. We were surprised to find that recombinant TxlA and S111980 lacking the C-terminal domain had reduced thioredoxin-like activity and were not stable in solution unless we added $50 \mathrm{mM}$ urea. CD spectroscopy revealed that the loss of the C-terminal domain reduced the secondary structure detected in the recombinant proteins, suggesting that the C-terminal domain is important for TxlA function because it stabilizes TxlA structure. 
We were unsuccessful (despite several attempts by different methods) in constructing site-directed mutants in the putative active site cysteine residues, so were unable to directly test the second part of this hypothesis. However, we performed extensive homology-based modeling of the predicted secondary and tertiary structure of TxlA and S111980. These models support the idea that the domains of TxlA and S111980 with sequence similarity to thioredoxins also fold like thioredoxins.

\section{TxlA is localized to the thylakoid membrane or intrathylakoid space by virtue of its $N$-terminal signal sequence.}

This hypothesis was tested by raising antibodies to the C-terminal domain of TxlA and using them to detect the presence of TxlA in appropriate subcellular fractions. We were concerned about the potential for cross-reactivity of antibodies with other thioredoxin-like proteins, so used a chemically synthesized polypeptide identical to the C-terminal domain rather than whole recombinant TxlA. The results indicate that TxlA is not soluble, and is localized to the thylakoid membrane.

\section{If TxlA is involved in a photosynthesis-specific process, it is essential for photoautotrophic growth but not for heterotrophic growth.}

This hypothesis was tested by constructing (or, more accurately, trying to construct) complete sll1980 (the txlA homologue) inactivation mutants in Synechocystis sp. strain PCC 6803, which unlike PCC 7942 is capable of mixotrophic (with glucose in the light), photoheterotrophic (with glucose in the light in the presence of DCMU) and chemoheterotrophic (with glucose in the dark except for a 15 minute pulse of light daily) growth. We were unable to generate sll1980 inactivation mutants under any growth conditions tested. This hypothesis has been refuted; S111980 is essential to PCC 6803 under photoautotrophic, mixotrophic, photoheterotrophic, and chemoheterotrophic growth conditions. If TxlA is involved in $b_{6} f$ complex synthesis, this result makes sense; both photosynthetic and respiratory electron transport in cyanobacteria require $b_{0} f$ while the $A$. thaliana HCF164- mutants could survive using the unaffected mitochondrial electron transport chain.

Interestingly, the S111980 inactivation merodiploids did have a pigmentation phenotype (high phycocyanin relative to chlorophyll) similar to the TxlXb phenotype when grown photoautotrophically. In addition, when grown heterotrophically they remained green instead turning of the golden color of wild-type cells, and showed enhanced sensitivity to the $b_{6} f$ complex inhibitor, DBMIB.

\section{If TxlA is involved in cytochrome biosynthesis, a difference in cytochrome complements will be detected in TxlA mutants.}

As mentioned under Hypothesis 3, we were unable to generate complete TxlAinactivated mutants in either PCC 6803 or PCC 7942, so were unable to test this hypothesis. We took an alternative approach by engineering a construct in PCC 7942 that expressed antisense $t x l A$ mRNA until the control of an IPTG-inducible promoter. Induction of antisense txlA was lethal, and we are still searching for growth conditions that will allow us to control the antisense mRNA expression well enough to exploit this mutant. We were not successful in producing a truncated S111980 mutant in PCC 6803 (purely for technical, not biological, reasons). 
We examined the cytochromes of the C-terminal truncation mutant $\mathrm{TxlXb}$ in PCC 7942 by both a cytochrome $f$-specific antibody and by heme staining. We were unable to detect any differences in the cytochromes of the TxlXb mutant versus wild-type cells. Reduced-minus-oxidized cytochrome absorbance spectra also revealed no differences. This result suggests that if TxlA is involved in $c$-type cytochrome biosynthesis, the $\mathrm{C}$-terminal truncated protein retains this function.

\section{TxlA interacts with at least one other gene or protein in performing its normal function.}

We have observed several instances in which the presence of truncated TxlA or S111980 appears to alter the phenotype of PCC 7942 or PCC 6803 even in the presence of normal copies of the $t x l A$ or sll1980 gene. (These include merodiploids of the inactivation mutants and the consistent phenotype of the $\mathrm{TxlXb}$ mutant from merodiploidy through homogeneity). These observations suggest that truncated TxlA or S111980 can interfere with the function of native TxlA or S111980 produced from wild type $t x l A$ or sll1980 gene copies, perhaps via dimerization or other protein-protein interactions.

We were unsuccessful in more directly testing this hypothesis by immunoprecipitations and cross-linking experiments. If TxlA interacts with other proteins via its Cterminal domain, it is possible the epitope(s) recognized by the antibody were inaccessible and that an alternate antibody will be required for these experiments. Efforts to determine the in vivo redox state of TxlA were also unsuccessful. Since we did not regenerate the TxlXg phenotype, we were unable to complement the mutation in search of interacting genes. Our results also indicate that the original phenotype might have been due to merodiploidy undetected by Southern blots.

The specific goals were:

\section{Establish PCC6803 txlA mutants and characterize them while also} extending the characterization of the original PCC7942 mutants.

We showed that the TxlA homologue in PCC 6803, S111980, is required for both photoautotropic and heterotrophic growth. This result is consistent with the suggestion from Arabidopsis thaliana that TxlA is required for the synthesis of $b_{6} f$ complex. PCC 6803 S111980 inactivation merodiploids have a phenotype similar to that of the PCC 7942 Cterminal domain truncation mutants (altered pigmentation), and are also more sensitive to the $b_{6} f$ complex inhibitor, DBMIB, than is wild type PCC 6803. The phenotype of these mutants is worth further investigation. Our PCC 7942 regulatable antisense TxlA mutants are also a promising avenue for future studies, once we can achieve better control.

Our characterization of the regenerated $\mathrm{TxlXb}$ (C-terminal truncation) mutants in $\mathrm{PCC}$ 7942 included monitoring pigmentation and growth rate under different light qualities and intensities and degrees of $\mathrm{CO}_{2}$-limitation, as well as extensive PAM fluorometry (which we chose instead of $77 \mathrm{~K}$ fluorescence spectroscopy because of the greater information it provides). These data have supported the original report of a subtle pigmentation phenotype (high phycocyanin to chlorophyll ratio), but have so far provided no new insights into the underlying defect caused by removal of the C-terminal domain. The phenotype of the TxlXb mutant is subtle, and the underlying defect remains elusive. However, we suggest that in addition to its essential function, TxlA has a regulatory function that is disrupted in the $\mathrm{TxlXb}$ mutants. 
2. Express and purify recombinant TxlA in E. coli, using the product to perform in vitro biochemical analysis of TxlA and to raise antibodies against TxlA.

The recombinant TxlA and S111980 studies established that both proteins have thioredoxin-like redox activity, and that the C-terminal domain is important in TxlA structure and function. We chose a different method to raise our antibodies.

\section{Biochemically characterize native TxlA in cyanobacteria by determining its subcellular localization and redox status. \\ 4. Apply both biochemical and genetic methods to identify other proteins and genes with which TxlA interacts.}

We found that TxlA, like HCF164, is anchored to the thylakoid membrane. Further progress toward achieving these two goals will require that we overcome a variety of technical issues, starting with the generation of alternative antibodies to support future studies.

\section{Summary}

We validated much of the previous work on TxlA in PCC 7942, although we have raised doubts about whether the original TxlXg phenotype was actually a homogeneous mutant. We showed that the TxlA homologue in PCC 6803, S111980, is required for both photoautotropic and heterotrophic growth. We found that TxlA is a membrane-associated protein, and that it has thioredoxin-like redox activity in vitro. Both the structure and redox function of TxlA are dependent on the C-terminal domain.

The results summarized here are described in greater detail in the Ph.D. thesis of Denise Kay, whose training was supported by this grant, and in the three manuscripts attached below.

Kay, D.M. 2004. A novel family of essential membrane-bound thioredoxin-like proteins in oxygenic photoautotrophs. Ph.D. Thesis, Rensselaer Polytechnic Institute.

Kay, D.M. and J.L. Collier. 2005. Homology models of the TxlA thioredoxin fold domain. To be submitted to BBA.

Kay, D.M., W.A. Williams, and J.L. Collier. 2005a. Synechocystis sp. strain PCC 6803 Sll1980 is an essential oxidoreductase. To be submitted to J. Bact.

Kay, D.M., W.A. Williams, and J.L. Collier. 2005b. The unique C-terminal domain is required for TxlA structure and function. To be submitted to J. Bact.

\section{References}

Collier, J.L. and A.R. Grossman. 1995. Disruption of a gene encoding a novel thioredoxinlike protein alters the cyanobacterial photosynthetic apparatus. J. Bact. 177(11):3269-3276.

Lennartz, K., H. Plucken, A. Seidler, P. Westhoff, N. Bechtold, and K. Meierhoff. 2001. HCF164 encodes a thioredoxin-like protein involved in the biogenesis of the cytochrome $\mathrm{b}_{6} \mathrm{f}$ complex in Arabidopsis. The Plant Cell 13:2539-2551. 